\title{
ON A THEOREM OF SCHWARZ TYPE FOR QUASICONFORMAL MAPPINGS IN SPACE
}

\author{
KAZUO IKOMA
}

To Professor Kryoshi Noshiro on the occasion of his 60 th birthday

A space ring $R$ is defined as a domain whose complement in the Moebius space consists of two components. The modulus of $R$ can be defined in variously different but essentially equivalent ways (see e.g. Gehring [3] and Krivov [5]), which is denoted by mod $R$. Following Gehring [2], we refer to a homeomorphism $y(x)$ of a space domain $D$ as a $K$-quasiconformal mapping, if the modulus condition

$$
\frac{1}{K} \bmod R \leqq \bmod y(R) \leqq K \bmod R
$$

is satisfied for all bounded rings $R$ with their closure $\bar{R} \subset D$, where $y(R)$ denotes the image of $R$ by $y=y(x)$. Then, it is evident that the inverse of a $K$-quasiconformal mapping is itself $K$-quasiconformal and that a $K_{1}$-quasiconformal mapping followed by a $K_{2}$-quasiconformal one is $K_{1} K_{2}$-quasiconformal. It is also well known that the restriction of a Moebius transformation to a space domain is equivalent to a 1-quasiconformal mapping of its domain.

The purpose of this paper is to prove Theorem 2 in the previous paper [4] (see also corrections to it added after the list of references in this paper) without the additional condition " $y(x)$ maps each radius of $|x|<1$ onto a curve which is normal to the image of each surface $|x|=r$ " and without the use of any isoperimetric inequality such as $A(r)^{3}-36 \pi V(r)^{2} \geqq 0$ used in its former proof, and to give the various space forms derived from there. All our arguments can be similarly carried over to higher dimensions, but we shall restrict ourselves for brebity sake to the Moebius 3-dimensional space.

1. First we enunciate the theorem.

THEOREM. Let $y=y(x)$ be a K-quasiconformal mapping of $|x|<1$ such that

Received February 4, 1966. 
$|y|<1$ and $y(0)=0$. Then it holds that

$$
\lim _{x \rightarrow 0} \inf _{x} \frac{|y(x)|}{|x|^{1 / K}} \leqq 1,
$$

where the equality holds if and only if $y(x)=f_{1 / K}\left(x\left(r, \theta_{1}, \theta_{2}\right)\right)$ :

$$
\left\{\begin{array}{l}
y_{1}=r^{1 / K} \cos \left(\theta_{1}+c_{1}\right), \\
y_{2}=r^{1 / K} \sin \left(\theta_{1}+c_{1}\right) \cos \left(\theta_{2}+c_{2}\right), \\
y_{3}=r^{1 / K} \sin \left(\theta_{1}+c_{1}\right) \sin \left(\theta_{2}+c_{2}\right),
\end{array}\right.
$$

$c_{1}, c_{2}$ being arbitrary real constants, which are uniquely determined except for rotations about the origin of $|y|<1$.

Hence, if two points given on $|x|=1$ correspond to two points given on $|y|=1$, for instance, the points $(1,0,0),(0,1,0)$ are carried into themselves respectively, the mapping obtained by putting $c_{1}=c_{1}=0$ in (1) is the only extremal one.

2. In proving this theorem, we use the following two lemmas.

Lemma 1. The modulus of a spherical ring bounded by two spherical surfaces with radii $a$ and $b(>a)$ is not greater than $\log \frac{b}{a}$. Further, its modulus attains the maximum value $\log \frac{b}{a}$ if and only if the spherical ring is concentric.

This is Lemma 3 proved in [4].

LEMMA 2. Let $y=y(x)$ be a K-quasiconformal mapping of $|x|<1$ onto $|y|<1$. If $y=y(x)$ maps $|x|=q$ for almost all $0<q<1$ onto $|y|=q^{1 / K}$, then it is nothing but the mapping $y=f_{1 / K}\left(x\left(r, \theta_{1}, \theta_{2}\right)\right)$ in the above Theorem.

Proof. First, we fix a system of cartesian coordinates $x_{1}, x_{2}, x_{3}$ to which corresponds polar coordinates $r, \theta_{1}, \theta_{2}$ such that $x_{1}=r \cos \theta_{1}, x_{2}=r \sin \theta_{1} \cos \theta_{2}, x_{3}=$ $r \sin \theta_{1} \sin \theta_{2}$ in the $x$-space. Similarly, introduce arbitrary systems of cartesian coordinates $y_{1}, y_{2}, y_{3}$ and the corresponding polar coordinates $\rho, \phi_{1}, \phi_{2}$ into the $y$. space. Then, the required mappings $y=y(x)$, can be represented by the form

$$
\left\{\begin{array}{l}
y_{1}=\rho \cos \left(\phi_{1}+c_{1}^{\prime}\right), \\
y_{2}=\rho \sin \left(\phi_{1}+c_{1}^{\prime}\right) \cos \left(\phi_{2}+c_{2}^{\prime}\right), \\
y_{3}=\rho \sin \left(\phi_{1}+c_{1}^{\prime}\right) \sin \left(\phi_{2}+c_{2}^{\prime}\right),
\end{array}\right.
$$

where each of $\rho, \phi_{1}, \phi_{2}$ is a function of three variables $r, \theta_{1}, \theta_{2}$ and $c_{1}^{\prime}, c_{2}^{\prime}$ are arbitrary real constants. From the assumption, there holds $\rho=r^{1 / K}$ for almost. 
all $r$ such that $0<r<1$.

Next, let $q$ be an arbitrary number satisfying the assumption and denote by $R$ the spherical ring $q^{1 / K}<|y|<1$. For the function $f(y)=\left(|y| \frac{1}{K} \log \frac{1}{q}\right)^{-1}$ in $R$, if we put

$$
S(\dot{R})=\iint_{|y|=r^{1 / K}} f(y)^{2} d \sigma
$$

and

$$
T(R)=\iiint_{R} f(y)^{3} d \tau
$$

where $d \sigma, d \tau$ denote the surface, volume element on $|y|=r^{1 / K}$, in $R$, respectively, then some simple calculations yield that

$$
S(R)=T(R)=\frac{4 \pi}{\left(\frac{1}{K} \log \frac{1}{q}\right)^{2}}
$$

Further, let $J(x)$ be the Jacobian of $y(x)$, and let $N(y)$ be the directional derivative of the inverse mapping $x=x(y)$ in the radial direction: $N(y)=$ $\lim _{k \rightarrow 0}\{x(y+k y)-x(y)\} / k y, k$ being real. Let $d \omega$ be the surface element on $|x|=r$, and denote by $\theta_{x}$ the angle between the radial ray and the inverse image vector $d x$ corresponding to the infinitesimal vector $d y$ in the radial direction. Then, through some geometric considerations, we obtain

$$
d \sigma=|J(x)||N(y)| \cos \theta_{x} d \omega .
$$

Hence it holds that

$$
S(R)=\iint_{|x|=r}|J(x)||N(y)| \cos \theta_{x}\left(r^{1 / K} \frac{1}{K} \log \frac{1}{q}\right)^{-2} d \omega .
$$

Applying Hölder's inequality, we have

$$
S(R)^{3,2} \leqq\left(\iint_{|x|=r} d \omega\right)^{1 / 2} \iint_{|x|=r}\left\{|J(x)||N(y)| \cos \theta_{x}\left(r^{1 / K} \frac{1}{K} \log \frac{1}{q}\right)^{-2}\right\}^{3 / 2} d \omega .
$$

Since $|N(y)| \leqq 1 / \min \frac{d y}{d x} \mid$ and $\left(|J(x)| / \min \left|\frac{d y}{d x}\right|^{3}\right)^{1 / 2} \leqq K$ almost everywhere (cf. Theorem 6.13 in Väisälä [6] or Theorem 4 in [2]), it follows from (2.2) that

$$
S(R)^{3 / 3} \leqq \sqrt{4 \pi} r K \iint_{|x|=r}\left(r^{1 / K} \frac{1}{K} \log \frac{1}{q}\right)^{-3}|J(x)| d \omega,
$$

so that 


$$
\frac{1}{\sqrt{4 \pi} r K} \leqq \frac{\iint_{|x|=r}\left(r^{1 / K} \frac{1}{K} \log \frac{1}{q}\right)^{-3}|J(x)| d \omega}{S(R)^{3 / 2}}
$$

By integrating with respect to $r$ on the interval $[q, 1]$, we have

$$
\frac{1}{K} \log \frac{1}{q} \leqq \sqrt{4 \pi} \cdot \frac{T(R)}{S(R)^{3 / 2}}
$$

However, the relation (2.1) implies that the equality holds in (2.3). Hence, the equality holds also in Hölder's inequality (2.2). Therefore, we have

$$
|J(x)||N(y)| \cos \theta_{x}=\frac{d \sigma}{d \omega}=\text { constant }
$$

for each fixed $r$ and almost all $\theta_{1}, \theta_{2}$. From the equality in (2.2), we have in fact

$$
\begin{aligned}
(4 \pi)^{3 / 2} & =(4 \pi)^{1 / 2} r^{1-3 / K} \iint_{|x|=r}\left(\frac{d \sigma}{d \omega}\right)^{3 / 2} d \omega \\
& =(4 \pi)^{1 / 2} r^{-2 / K} \iint_{|y|=r 1 / K} r^{1-1 / K}\left(\frac{d \sigma}{d \omega}\right)^{1 / 2} d \sigma
\end{aligned}
$$

so that

$$
\iint_{|y|=r^{1 / K}}\left\{r^{1-1 / K}\left(\frac{d \sigma}{d \omega}\right)^{1 / 2}-1\right\} d \sigma=0
$$

It is written in the form

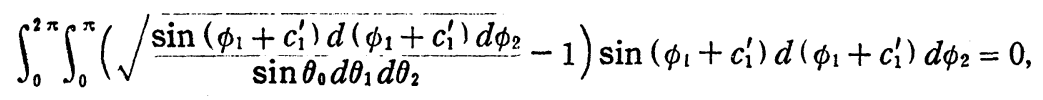

since $\frac{d \sigma}{d \omega}=r^{2 / K} \sin \left(\phi_{1}+c_{1}^{\prime}\right) d\left(\phi_{1}+c_{1}^{\prime}\right) d \phi_{2} / r^{2} \sin \theta_{1} d \theta_{1} d \theta_{2}$. Hence it follows that

$$
\frac{\sin \left(\phi_{1}+c_{1}^{\prime}\right) d\left(\phi_{1}+c_{1}^{\prime}\right) d \phi_{2}}{\sin \theta_{1} d \theta_{1} d \bar{\theta}_{2}}=1
$$

for almost all $0<r<1$.

This implies that for almost all $0<r<1$, the surface element at $P\left(r, \theta_{1}, \theta_{2}\right)$ on $|x|=r$ is equal to the one at the corresponding point $Q\left(r, \phi_{1}+c_{1}^{\prime}, \phi_{2}+c_{2}^{\prime}\right)$ on $|y|=r$. As is easily seen, the set on $|x|=r($ resp. $|y|=r)$ such that the surface element at each point of its set equals to the one at $P$ (resp. $Q$ ) is the circle on $|x|=r$ (resp. $|y|=r$ ) with the same $\theta_{1}$ (resp. $\phi_{1}+c_{1}^{\prime}$ ) as in $P$ (resp. $Q$ ). Therefore, we have $d \phi_{2} / d \theta_{2}=k$ (constant). Integrating it over $[0,2 \pi]$, we have 
$k=1$, and so $\phi_{2}=\theta_{2}+c_{2}$, where $c_{2}$ is any real constant. Then, it follows from $\sin \left(\phi_{1}+c_{1}^{\prime}\right) d\left(\phi_{1}+c_{1}^{\prime}\right)=\sin \theta_{1} d \theta_{1}$ that $\phi_{1}+c_{1}^{\prime}=\theta_{1}$ or $\phi_{1}=\theta_{1}+c_{1}$, where $c_{1}=-c_{1}^{\prime}$. That is to say, we have found that for almost all $0<r<1, \rho=r^{1 / K}, \phi_{1}=\theta_{1}+c_{1}$ and $\phi_{2}=\theta_{2}+c_{2}, c_{1}, c_{2}$ being arbitrary real constants. Since $y=y(x)$ is a homeomorphism, it must be the mapping in (1). Conversely, it is quite clear that $y=f_{1 / K}\left(x\left(r, \theta_{1}, \theta_{2}\right)\right)$ satisfies the assumption in Lemma 2.

3. Proof of Theorem. Let $V(r)$ be the volume bounded by the image $y(|x|=r)$ of $|x|=r(<1)$ under $y=y(x)$, and denote by $y\left(r<|x|<r^{\prime}\right)$ the image of $r<|x|<r^{\prime}$ under $y=y(x)$. Then, by an extension of Golusin's theorem (see Theorem 3 in Gehring $[1]$ ), we have

$$
\bmod y\left(r<|x|<r^{\prime}\right) \leqq \frac{1}{3} \log \frac{V\left(r^{\prime}\right)}{V(r)} .
$$

Together with the inequality

$$
\frac{1}{K} \log \frac{r^{\prime}}{r} \leqq \bmod y\left(r<|x|<r^{\prime}\right)
$$

followed from the one sided modulus condition in $(*)$, we get

$$
\frac{V(r)}{r^{3 / K}} \leqq \frac{V\left(r^{\prime}\right)}{\left(r^{\prime}\right)^{3 / K}}
$$

Since this shows that $V(r) / r^{n / K}$ is a non-decreasing function in $r$, we have

$$
\frac{V(r)}{r^{3 / K}} \leqq V(1)=\frac{4}{3} \pi
$$

Put $\min _{|x|=r}|y(x)|=m(r)$. Then it is obvious from $y(0)=0$ that

$$
\frac{4}{3} \pi(m(r))^{3} \leqq V(r)
$$

Thus we obtain

$$
\liminf _{x \rightarrow 0} \frac{|y(x)|}{\mid x i^{1 / K}}=\lim _{r \rightarrow 0} \inf \frac{m(r)}{r^{1 / K}} \leqq \lim _{r \rightarrow 0} \inf \left\{\frac{3 V(r)}{4 \pi r^{3 / K}}\right\}^{1 / 3} \leqq 1 .
$$

Now, suppose that $y=y(x)$ satisfying the assumption of the theorem induces the signs of equality in (3.3). Then, the signs of equality hold for almost all $r(0<r<1)$ in $(3.2)$ and (3.1). Since $V(r) / r^{3 / \kappa}$ was a non-decreasing function in $r$, it is easy to see that the sign of equality for all $r$ holds in (3.1), i.e.

$$
V(r)=\frac{4}{3}-\pi r^{3 / K}
$$


From this and the equality in (3.2), we have

$$
m(r)=\sqrt[3]{\frac{3 V(r)}{4 \pi}}=r^{1 / K}
$$

for almost all $r$. Hence it follows that the image $y(|x|=r)$ for almost all $r$ is a spherical surface with radius $r^{1 / K}$. Here, by making $r \rightarrow 1$, it follows also that $y=y(x)$ maps $|x|<1$ onto $|y|<1$. Accordingly, as is seen by Lemma 1 , the modulus of the spherical ring $y(r<|x|<1)$ for almost all $r$ is not greater than $\frac{1}{K} \log \frac{1}{r}$. while its modulus is not less than $\frac{1}{K} \log \frac{1}{r}$ in view of the modulus condition $(*)$. Therefore it follows that its modulus is equal to $\frac{1}{K} \log \frac{1}{r}$. Then, by Lemma 1 again, we can see that the center of $y(|x|=r)$ for almost all $r$ is the origin $y=0$. Thus we conclude from Lemma 2 that the required extremal mapping $y(x)$ is nothing but $f_{1 / K}\left(x\left(r, \theta_{1}, \theta_{2}\right)\right)$ in (1).

4. In order to obtain the corresponding estimates in the case where the restriction $y(0)=0$ is removed, we use the following lemma.

Lemma 3. A Moebius transformation i.e. 1-quasiconformal mapping $X=X(\xi)$ of $|\xi|<1$ onto $|X|<1$ which carries $\xi_{a}=(a, 0,0)$ into the origin $(0,0,0)$ is given by

$$
\left\{\begin{array}{l}
X_{1}=-\frac{\left(\frac{1}{a^{2}}-1\right)\left(\xi_{1}-\frac{1}{a}\right)}{\left(\xi_{1}-\frac{1}{a}\right)^{2}+\xi_{2}^{2}+\xi_{3}^{2}}-\frac{1}{a} \\
X_{2}=\frac{\left(\frac{1}{a^{2}}-1\right) \xi_{2}}{\left(\xi_{1}-\frac{1}{a}\right)^{2}+\xi_{2}^{2}+\xi_{3}^{2}} \\
X_{3}=\frac{\left(\frac{1}{a^{2}}-1\right) \xi_{3}}{\left(\xi_{1}-\frac{1}{a}\right)^{2}+\xi_{2}^{2}+\xi_{3}^{2}}
\end{array}\right.
$$

which is simply denoted by $X=X(\xi)$.

Proof. Some elementary computations using the cross ratio yields that the linear transformation

$$
X_{1}+i X_{2}=\frac{a-\left(\xi_{1}+i \xi_{2}\right)}{a\left(\xi_{1}+i \xi_{2}\right)-1}=-\frac{\left(\frac{1}{a^{2}}-1\right)\left(\xi_{1}-\frac{1}{a}-i \xi_{2}\right)}{\left(\xi_{1}-\frac{1}{a}\right)^{2}+\xi_{2}^{2}}-\frac{1}{a}
$$


carries the disc $\sqrt{\xi_{1}^{2}+\xi_{2}^{2}}<1$ into $\sqrt{X_{1}^{2}+X_{2}^{2}}<1$ and $\left(\xi_{1}, \xi_{2}\right)=(a, 0)$ into $\left(X_{1}, X_{2}\right)$ $=(0,0)$. We shall denote this linear transformation by $X_{1}+i X_{2}=f\left(\xi_{1}+i \xi_{2}\right)$.

Next, we denote by $\left(\xi_{1}, s, \varphi\right)$ and $\left(X_{1}, t, \psi\right)$ the semi-polar coordinates of $\left(\xi_{1}, \xi_{2}, \xi_{3}\right)$ and $\left(X_{1}, X_{2}, X_{3}\right)$, respectively. Consider the linear transformation $X_{1}+i t=f\left(\xi_{1}+i s\right) \cdot$ which carries the intersection of each plane $\varphi=u(0 \leqq u \leqq 2 \pi)$ with the sphere $|\xi|<1$ into the intersection of each plane $\psi=u(0 \leqq u \leqq 2 \pi)$ with the sphere $|X|<1$, where $\psi$ takes the same $u$ as each $u$ taken by $\varphi$. Then, such a linear transformation produces immediately our required mapping.

5. Now, let $y_{0}$ be $y\left(x_{0}\right)$ for any $x_{0}$ in $|x|<1$. We denote by $\xi=\xi(x)$ the rotation about the origin which carries $|x|<1$ into $|\xi|<1$ and $x=x_{0}$ into $\xi_{\left|x_{0}\right|}=$ $\left(\left|x_{0}\right|, 0,0\right)$, and let $\eta=\eta(y)$ be the similar rotation which carries $|y|<1$ into $|\eta|<1$ and $y=y_{0}$ into $\eta_{\left|y_{0}\right|}=\left(\left|y_{0}\right|, 0,0\right)$. Further, let $X=X(\xi)$ be the Moebius transformation obtained by putting $a=\left|x_{0}\right|$ in (4), and let $Y=Y(\eta)$ be the similar one obtained by replacing $X, \xi, a$ in (4) with $Y, \eta,\left|y_{0}\right|$, respectively. Then we have the following corollaries.

Corollary 1. Let $y=y(x)$ be a K-quasiconformal mapping of $|x|<1$ such that $|y(x)|<1$. Then, for any $x_{0}$ in $|x|<1$, it holds that

$$
\liminf _{x \rightarrow x_{0}} \frac{\left|y(x)-y\left(x_{0}\right)\right|}{\left|x-x_{0}\right|^{1 / K}} \leqq \frac{1-\left|y\left(x_{0}\right)\right|^{2}}{\left(1-\left|x_{0}\right|^{2}\right)^{1 / K}} .
$$

The sign of equality holds if and only if $y(x)=g_{1 / K}(x)$, where $g_{1 / K}(x)$ is the composite mapping of the above $\xi=\xi(x), X=X(\xi), Y=Y(\eta), \eta=\eta(y)$ and the quasiconformal mapping $Y=F_{1 / K}(X)$ having the same form as (1).

Proof. First, consider the composite mapping of four Moebius transformations $\xi=\xi(x), X=X(\xi), \eta=\eta(y)$ and $Y=Y(\eta)$ mentioned above and a $K$ quasiconformal mapping $y=y(x)$ in this corollary. Then, the restriction $Y=$ $Y(X)$ of such a composite mapping to $|X|<1$ maps $|X|<1 K$-quasiconformally onto $|Y|<1$ and carries the origin into itself, and so $Y=Y(X)$ satisfies the assumption of Theorem in $\$ 1$.

Next, simple computations for $X=X(\xi)$ yield that

$$
|X|=\frac{\sqrt{\left(\xi_{1}-\left|x_{0}\right|\right)^{2}+\xi_{2}^{2}+\xi_{3}^{2}}}{\sqrt{\left(\left|x_{0}\right| \xi_{1}-1\right)^{2}+\left(\left|x_{0}\right| \xi_{2}\right)^{2}+\left(\left|x_{0}\right| \xi_{3}\right)^{2}}}=\frac{|\xi-\xi| x_{0} \mid}{\left|x_{0}\right| \mid \xi-\frac{\xi_{\left|x_{0}\right|} \mid}{\left|x_{0}\right|^{2}}} .
$$

Similarly, we have for $Y=Y(\eta)$, 


$$
|Y|=\frac{|\eta-\eta| y_{0} \mid}{\left|y_{0}\right|\left|\eta-\frac{\eta_{\left|y_{0}\right|} \mid}{\left|y_{0}\right|^{2}}\right|}
$$

Further, the distance between two points is invariant under any rotation about the origin. Hence we find for the above rotations $\xi=\xi(x)$ and $\eta=\eta(y)$. that

$$
\begin{aligned}
& \left|\xi-\xi_{\left|x_{0}\right|}\right|=\left|x-x_{0}\right|,\left|\xi-\frac{\xi_{\left|x_{0}\right|} \mid}{\left|x_{0}\right|^{2} \mid}\right|=\mid x-\frac{x_{0} \mid}{\left|x_{0}\right|}, \\
& \left|\eta-\eta_{\left|y_{0}\right|}\right|=\left|y-y_{0}\right| \text { and }\left|\eta-\frac{\eta_{\left|y_{0}\right|} \mid}{\left|y_{0}\right|^{2} \mid}=\right| y-\frac{y_{0}}{\left|y_{0}\right|} \mid .
\end{aligned}
$$

Thus, we have finally

$$
|X|=\frac{\left|x-x_{0}\right|}{\left|x_{0}\right|\left|x-\frac{x_{0}}{\left|x_{0}\right|^{2}}\right|},|Y|=\frac{\left|y-y_{0}\right|}{\left|y_{0}\right|\left|y-\frac{y_{0}}{\left|y_{0}\right|^{2}}\right|} .
$$

Consequently, it can be deduced from Theorem in $\S 1$ that

$$
\liminf _{x \rightarrow 0} \frac{|Y|}{|X|^{1 / K}}=\liminf _{x \rightarrow x_{0}} \frac{\left|y-y_{0}\right|}{\left|x-x_{0}\right|^{1 / K}} \cdot \frac{\left(\left|x_{0}\right|\left|x-\frac{x_{0}}{\left|x_{0}\right|^{2}}\right|\right)^{1 / K}}{\left|y_{0}\right|\left|y-\frac{y_{0}}{\left|y_{0}\right|^{2}}\right|} \leqq 1,
$$

so that we have

$$
\liminf _{x \rightarrow x_{0}} \frac{\left|y-y_{0}\right|}{\left|x-x_{0}\right|^{1 / K}} \leqq \lim _{x \rightarrow x_{0}} \frac{\left|y_{0}\right|\left|y-\frac{y_{0}}{\left|x_{0}\right|^{2}}\right|}{\left(\left|x_{0}\right| \mid x-\frac{x}{\left|x_{0}\right|^{2} \mid}\right)^{1 / K}}=\frac{1-\left|y_{0}\right|^{3}}{\left(1-\left|x_{0}\right|^{2}\right)^{2 / K}} \text {, q.e.d. }
$$

It is quite easy to see that the extremal mapping getting the equality here to hold is nothing but the required $y=g_{1 / K}(x)$.

Considering the inverse of a mapping in Corollary 1 , we have

Corollary 1.1. Let $y=y(x)$ be a K-quasiconformal mapping of a domain in $|x|<1$ onto $|y|<1$. Then, for any $x_{0}$ in $|x|<1$, there holds

$$
\limsup _{x \rightarrow x_{0}} \frac{\left.\mid y(x)-y^{\prime} x_{0}\right) \mid}{\left.\left|x-x_{0}\right|\right|^{K}} \geqq \frac{1-\left|y\left(x_{0}\right)\right|^{2}}{\left(1-\left|x_{0}\right|^{2}\right)^{K}} \text {. }
$$

The sign of equality holds if and only if $y=y(x)$ is the composite mapping of the same $\xi=\xi(x), X=X(\xi), Y=Y(y), \eta=\eta(y)$ as in Corollary 1 and $Y=F_{K}(X)$ replacing $\frac{1}{K}$ in $Y=F_{1 / K}(X)$ of Corollary 1 with $K$.

The following is an immediate consequence of Corollaries 1 and 1.1. 
Corollary 1.2. Let $y=y(x)$ be a K-quasiconformal mapping of $|x|<1$ onto $|y|<1$. Then, for any $x_{0}$ in $|x|<1$ and $k \geqq K$, it holds that

$$
\liminf _{x \rightarrow x_{0}} \frac{\left|y(x)-y\left(x_{0}\right)\right|}{\left|x-x_{0}\right|^{1 / k}} \leqq \frac{1-\left|y\left(x_{0}\right)\right|^{2}}{\left(1-\left|x_{0}\right|^{2}\right)^{1 / k}} \leqq \frac{1-\left|y\left(x_{0}\right)\right|^{2}}{\left(1-\left|x_{0}\right|^{2}\right)^{k}} \leqq \limsup _{x \rightarrow x_{0}} \frac{\left|y(x)-y\left(x_{0}\right)\right|}{\left|x-x_{0}\right|^{k}} .
$$

6. We prove the following lemma necessary in the case where the sphere is replaced by the half-space.

Lemma 4. A 1-quasiconformal mapping of the half-space $x_{1}>0$ onto the unit sphere $|X|<1$ carrying $x_{a}=\left(a_{1}, a_{2}, a_{3}\right)$ in the half-space $x_{1}>0$ into the origin is given by

$$
\left\{\begin{aligned}
X_{1} & =\frac{-2 a_{1}\left(x_{1}+a_{1}\right)}{\left(x_{1}+a_{1}\right)^{2}+\left(x_{2}-a_{2}\right)^{2}+\left(x_{3}-a_{3}\right)^{2}}+1 \\
X_{2} & =\frac{2 a_{1}\left(x_{2}-a_{3}\right)}{\left(x_{1}+a_{1}\right)^{2}+\left(x_{2}-a_{2}\right)^{2}+\left(x_{3}-a_{3}\right)^{2}} \\
X_{3} & =\frac{2 a_{1}\left(x_{3}-a_{3}\right)}{\left(x_{1}+a_{1}\right)^{2}+\left(x_{2}-a_{2}\right)^{2}+\left(x_{3}-a_{3}\right)^{2}}
\end{aligned}\right.
$$

which is simply denoted by $X=X(x)$.

Proof. First, perform the translation $\xi_{1}=x_{1}, \xi_{2}=x_{2}-a_{2}, \xi_{3}=x_{3}-a_{3}$ which carries $x_{a}=\left(a_{1}, a_{2}, a_{3}\right)$ into $\xi_{a}=\left(a_{1}, 0,0\right)$. Next, we make easily the linear transformation

$$
X_{1}+i X_{2}=\frac{-2 a_{1}\left(\xi_{1}+a_{1}\right)}{\left(\xi_{1}+a_{1}\right)^{2}+\xi_{2}^{2}}+1+\frac{2 a_{1} \xi_{2} i}{\left(\xi_{1}+a_{1}\right)^{2}+\xi_{2}^{2}}
$$

carrying the half-plane $\xi_{1}>0, \xi_{3}=0$ into the unit $\operatorname{disc} \sqrt{X_{1}^{2}+X_{2}^{2}}<1, X_{3}=0$ and $\left(\xi_{1}, \xi_{2}\right)=\left(a_{1}, 0\right)$ into $\left(X_{1}, X_{2}\right)=(0,0)$. Hereafter, by the similar process as in the proof of Lemma 3 , we have the required mapping.

7. By the aid of Lemma 4, we have the following half-space forms of Schwarz type.

COROLlaRY 2. Let $y=y(x)$ be a K-quasiconformal mapping of the half-space $x_{1}>0$ into $y_{1}>0$. Then, for any $x_{a}=\left(a_{1}, a_{2}, a_{3}\right)$ in $x_{1}>0$

$$
\liminf _{x \rightarrow x_{i}} \frac{\left|y(x)-y\left(x_{a}\right)\right|}{\left|x-x_{a}\right|^{1 / K}} \leqq \frac{2\left(y_{1} \text {-component of } y\left(x_{a}\right)\right)}{\left\{2\left(x_{1} \text {-component of } x_{a}\right)\right\}^{1 / K}} .
$$

The sign of equality holds if and only if $y(x)=h_{1 / K}(x)$, where $h_{1 / K}(x)$ is the composite mapping of $X=X(x)$ in (6) and $Y=Y(y)$ replacing $X, x, x_{a}$ in (6) with 
$Y, y, y\left(x_{a}\right)$, respectively, and the mapping $Y=F_{1 / K}(X)$ mentioned in Corollary 1.

Proof. Consider the composite mapping of $X=X(x), Y=Y(y)$ and a mapping $y=y(x)$ in this corollary. Then its restriction $Y=Y(X)$ to $|X|<1$ maps $|X|<1 K$-quasiconformally into $|Y|<1$ and carries the origin into itself. Hence $Y=Y(X)$ satisfies the assumption of Theorem in $\S .1$.

After some computations for the absolute value of $X=X(x)$, we obtain

$$
|X|=\sqrt{\frac{\left|x-x_{a}\right|^{2}\left|x-\bar{x}_{a}\right|^{2}}{\left|x-\bar{x}_{a}\right|^{4}}}=\frac{\left|x-x_{a}\right|}{\left|x-\bar{x}_{a}\right|}
$$

where $\bar{x}_{a}$ denotes the symmetric point of $x_{a}$ with respect to the plane $x_{1}=0$. Just similarly, we have

$$
|Y|=\frac{\left|y-y\left(x_{a}\right)\right|}{\left|y-y\left(x_{a}\right)\right|}
$$

$\overline{y\left(x_{a}\right)}$ being the symmetric point of $y\left(x_{a}\right)$ with respect to the plane $y_{1}=0$.

Then, we conclude by Theorem in $\S 1$ that

$$
\liminf _{x \rightarrow 0} \frac{|Y(X)|}{|X|^{1 / K}}=\liminf _{x \rightarrow x_{a}} \frac{\left|y(x)-y\left(x_{a}\right)\right|}{\mid y(x)-\overline{y\left(x_{a}\right) \mid}}\left(\frac{\left|x-\bar{x}_{a}\right|}{\left|x-x_{a}\right|}\right)^{1 / K} \leqq 1,
$$

so that we have

$$
\liminf _{x \rightarrow x_{a}} \frac{\left|y(x)-y\left(x_{a}\right)\right|}{\left|x-x_{a}\right|^{1 / K}} \leqq \lim _{x \rightarrow \infty a} \frac{\left|y(x)-y\left(x_{a}\right)\right|}{\left|x-\bar{x}_{a}\right|^{1 / K}}=\frac{2\left(y_{1}-\text { component of } y\left(x_{a}\right)\right)}{\left\{2\left(x_{1}-\text { component of } x_{a}\right)\right\}^{1 / K}} .
$$

It is easy to verify that the extremal mapping is nothing but $y=h_{1 / K}(x)$ as required.

Considering the inverse of a mapping in Corollary 2, we bave immediately

Corollary 2.1. Let $y=y(x)$ be a K-quasiconformal mapping of a domain in $x_{1}>0$ onto $y_{1}>0$. Then, for any $x_{a}$ in $x_{1}>0$, there holds

$$
\limsup _{a \rightarrow x_{a}} \frac{\left|y(x)-y\left(x_{a}\right)\right|}{\left|x-x_{a}\right|^{K}} \geqq \frac{2\left(y_{1}-\text { component of } y\left(x_{a}\right)\right)}{\left\{2\left(x_{1}-\text { component of } x_{a}\right)\right\}^{K}} \text {. }
$$

The sign of equality holds if and only if $y=y(x)$ is the composite mapping of $X=X(x), Y=Y(y)$ mentioned in Corollary 2 and the mapping $Y=F_{K}(X)$ mentioned in Corollary 1.1.

The following is an immediate consequence of Corollaries 2 and 2.1.

Corollary 2.2. Let $y=y(x)$ be a K-quasiconformal mapping of $x_{1}>0$ onto 
$y_{1}>0$. Then, for any $k \geqq K$ and $x_{a}$ in $x_{1}>0$ (whose $x_{1}$-component is not greater than $1 / 2$ ), it holds that

$$
\begin{aligned}
& \liminf _{x \rightarrow x_{a}} \frac{\left|y(x)-y\left(x_{a}\right)\right|}{\left|x-x_{a}\right|^{1 / k}} \leqq \frac{2\left(y_{1}-\text { component of } y\left(x_{a}\right)\right)}{\left\{2\left(x_{1}-\text { component of } x_{a}\right)\right\}^{1 / k}} \\
& (\leqq) \frac{2\left(y_{1}-\text { component of } y\left(x_{a}\right)\right)}{\left\{2\left(x_{1}-\text { component of } x_{a}\right)\right\}^{k}} \leqq \limsup _{x \rightarrow x_{a}} \frac{\left|y(x)-y\left(x_{a}\right)\right|}{\left|x-x_{a}\right|^{k}},
\end{aligned}
$$

where the condition for the $x_{1}$-component of $x_{a}$ in ( ) and the sign $\leqq$ in ( ) marked above are simultaneous.

Remark. Under the same condition as Theorem in $\S 1$, another theorem of Schwarz type " $\left\{\emptyset_{3}\left(\frac{1}{|x|}\right)\right\}^{1 / K} \leqq \emptyset_{3}\left(\frac{1}{|y|}\right)$ holds for any $0<|x|<1$ " has been established. (Theorem 1 in [4]). Therefore, if we use this result together with the relations (5) in $\S 5$ and $(7.1),(7.2)$ in $\S 7$, then other corollaries of Schwarz type under the same conditions as Corollaries 1 and 2 and etc., respectively, will be found. However, it is still open for us whether or not they are extended to higher dimensions and the extremal quasiconformal mapping in them exists.

\section{REFERENCES}

[1] Gehring, F. W., Symmetrization of rings in space, Trans. Amer. Math. Soc., 101 (1961), 499-516.

[2] - Rings and quasiconformal mappings in space. ibid., 103 (1962), 353-393.

[3] -- Extremal length definitions for the comformal capacity of rings in space, Mich. Math. J., 9 (1962), 137-150.

[4] Ikoma, K., On the distortion and correspondence under quasiconformal mappings in space, Nagoya Math. J., 25 (1965), 175-203.

[ 5 ] Krivov, V. V., Some properties of moduli in space, Dokl. Acad. Nauk SSSR 154 (1964), 510-513 (Soviet Math. Dokl. 5 (1964), 113-117).

[6] Väisälä, J., On quasiconformal mappings in space, Ann. Acad. Sci. Fenn., A. I. 298 (1961), 1-36.

Department of Mathematics, Yamagata University 
Added. Corrections to [4]:

p. 179 , line 5 from below, for " $y_{j}=$ " read " $Y_{j}=$ ".

p. 181, lines 11, 12 from below, for " $y_{1}=r^{1 / K} \cos \theta_{1}, y_{2}=r^{1 / K} \sin \theta_{1} \cos \left(\theta_{2}+c\right)$, $y_{3}=r^{1 / \pi} \sin \theta_{1} \sin \left(\theta_{2}+c\right)$ with a real constant $c$ " read " $y_{1}=r^{1 / k} \cos \left(\theta_{1}+c_{1}\right), y_{2}=r^{1 / k}$ $\sin \left(\theta_{1}+c_{1}\right) \cos \left(\theta_{2}+c_{2}\right), \quad y_{3}=r^{1 / K} \sin \left(\theta_{1}+c_{1}\right) \sin \left(\theta_{2}+c_{2}\right)$ with arbitrary real constants $c_{1}, c_{2} "$.

p. 183, line 1, for " $y_{1}=\rho \cos \phi_{1}, y_{2}=\rho \sin \phi_{1} \cos \phi_{2}, y_{3}=\rho \sin \phi_{1} \sin \phi_{2}$ " read “ $y_{1}=\rho \cos \left(\phi_{1}+c_{1}^{\prime}\right), \quad y_{2}=\rho \sin \left(\phi_{1}+c_{1}^{\prime}\right) \cos \left(\phi_{2}+c_{2}^{\prime}\right), \quad y_{3}=\rho \sin \left(\phi_{1}+c_{1}^{\prime}\right) \sin \left(\phi_{2}+c_{2}^{\prime}\right)$, $c_{1}^{\prime}, c_{2}^{\prime}$ being arbitrary real constants".

p. 183 , line 4 , for " $|J(x)| /|N(x)|=\sin \phi_{1} d \phi_{1} d \phi_{2} / \sin \theta_{1} d \theta_{1} d \theta_{2}=$ constant" read " $|J(x)| /|N(x)|=$ constant and $\sin \left(\phi_{1}+c_{1}^{\prime}\right) d\left(\phi_{1}+c_{1}^{\prime}\right) d \phi_{2} / \sin \theta_{1} d \theta_{1} d \theta_{2}=$ constant".

p. 183 , lines 7, 9 and 11 , for " $\sin \phi_{1} d \phi_{1} d \phi_{2}$ " read " $\sin \left(\phi_{1}+c_{1}^{\prime}\right) d\left(\phi_{1}+c_{1}^{\prime}\right) d \phi_{2}$ ".

p. 183 , line 14 , for " $\theta_{1}=\phi_{1}$ " read " $\phi_{2}=\theta_{2}+c_{2}$ ".

p. 183 , line 15 , for " $d \phi_{2}=d \theta_{2}$, so that $\phi_{2}=\theta_{2}+c, c$ being any real constant" read " $\sin \left(\phi_{1}+c_{1}^{\prime}\right) d\left(\phi_{1}+c_{1}^{\prime}\right)=\sin \theta_{1} d \theta_{1}$, so that $\phi_{1}=\theta_{1}+c_{1}, c_{1}, c_{2}$ being arbitrary real constants". 